operation characteristic (ROC) curves were performed to evaluate the discriminative utility of these parameters for disease activity of axSpA. Furthermore, the evaluation of the risk factors of axSpA was conducted using binary logistic regression analysis.

Results: CAR, ESR, CRP, NLR, PLR and MLR in axSpA patients were significantly higher than those in the control group $(p<0.05$ for each), while ALB was significantly lower $(p<0.001)$. Similarly, CAR in remission group was higher than that in control group $(p<0.001)$ and was lower than that in active group $(p<0.001)$. Besides, there were significantly positive correlations between CAR and ESR $(r=0.702, P<0.001)$, CRP $(r=0.996, P<0.001)$, BASDAI $(r=0.329, p<0.001)$ and BASFI $(r=0.328, P<0.001)$. Furthermore, ROC suggested that the area under the curve (AUC) of CAR was 0.701 , which was the highest. The optimal cutoff point of CAR was 0.3644 , with sensitivity and specificity of $58.5 \%$ and $79.0 \%$. Logistic analysis results revealed that elevated CAR and MLR were independent risk factors for axSpA (EXP $(B)=15.546,95 \% C l: 5.898-40.979, P<0.001$; EXP $(B)=2.206,95 \% C l: 1.077-$ 4.519, $\mathrm{P}=0.031$, respectively).

Conclusion: CAR was increased in axSpA patients especially in active group, and significantly correlated with disease activity. CAR may serve as a novel inflammatory marker of monitoring disease activity in patients with axSpA.

References:

[1] He, Y., et al., Correlation between albumin to fibrinogen ratio, C-reactive protein to albumin ratio and Th17 cells in patients with rheumatoid arthritis. Clin Chim Acta, 2020. 500: p. 149-154.

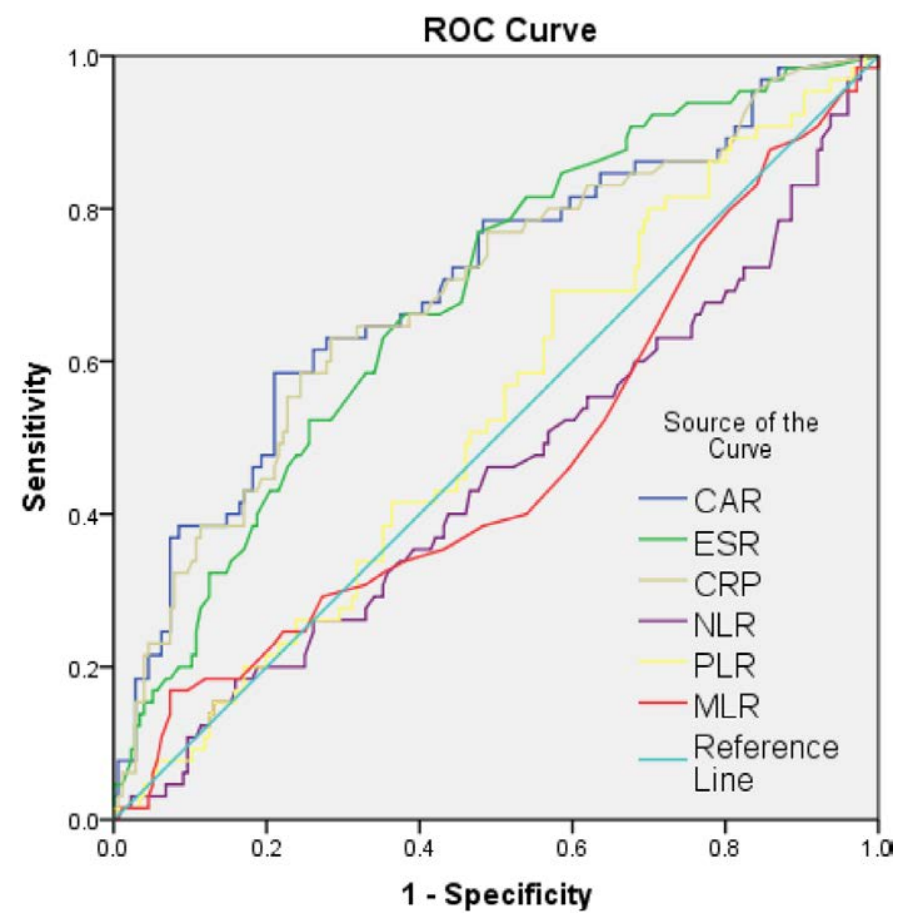

Fig 1. ROC curve analysis of the discriminative values of the parameters for disease activity of axSpA

Table 1. Discriminative values of the parameters for disease activity of axSpA

\begin{tabular}{lccccc}
\hline & AUC & $95 \% \mathrm{Cl}$ & Optimal cutoff point & Specificity & Sensitivity \\
\hline CAR & 0.701 & $0.623-0.778$ & 0.3644 & $79.0 \%$ & $58.5 \%$ \\
NLR & 0.450 & $0.365-0.534$ & 3.165 & $84.1 \%$ & $18.5 \%$ \\
PLR & 0.528 & $0.448-0.608$ & 127.385 & $42.6 \%$ & $69.2 \%$ \\
MLR & 0.468 & $0.384-0.553$ & 0.385 & $92.6 \%$ & $16.9 \%$ \\
ESR & 0.685 & $0.612-0.758$ & 15.5 & $52.3 \%$ & $76.9 \%$ \\
CRP & 0.691 & $0.614-0.769$ & 10.85 & $71.6 \%$ & $63.1 \%$ \\
\hline
\end{tabular}

CAR, C-reactive protein to albumin ratio; NLR, neutrophil-lymphocyte ratio; PLR, platelet-lymphocyte ratio; MLR, monocyte-lymphocyte ratio; CRP, C reactive protein; ESR, erythrocyte sedimentation rate; AUC, areas under the ROC curve

Disclosure of Interests: None declared DOI: 10.1136/annrheumdis-2020-eular.5199

\section{SAT0380 ENHANCING RHEUMATOLOGY REFERRALS AMONG INFLAMMATORY BOWEL DISEASE PATIENTS WITH SUSPECTED AXIAL SPONDYLOARTHRITIS}

C. S. E. Lim ${ }^{1}$, M. Tremelling ${ }^{2}$, L. Hamilton ${ }^{1}$, A. Macgregor ${ }^{1}$, K. Gaffney ${ }^{1} .{ }^{1}$ Norfolk and Norwich University Hospital, Rheumatology, Norwich, United Kingdom;

${ }^{2}$ Norfolk and Norwich University Hospital, Department of Gastroenterology, Norwich, United Kingdom

Background: Axial spondyloarthritis (axSpA) is associated with inflammatory bowel disease (IBD). In IBD patients, the clinical probability of axSpA increases in those with chronic back pain (CBP) whose symptoms started before the age of forty-five years old. In practice, this should trigger a rheumatology review especially if accompanied by other symptoms suspicious of inflammatory disease. However, in any health system, the goal of identifying all possible cases need to be balanced with the practical realisation of the finite resources available.

Objectives: The study aimed to define the clinical characteristics of a subgroup of IBD patients who are routinely managed in secondary care who have an increased clinical probability for axSpA. Identification of these characteristics may help improve the quality and specificity of referrals to Rheumatology from Gastroenterology clinics.

Methods: An analytical cross-sectional study was undertaken. Consecutive IBD patients attending routine Gastroenterology clinics were sent a modified validated back pain questionnaire. The questionnaire included the presence or absence of a previous diagnosis of axSpA; components of validated inflammatory back pain criteria; diagrams to indicate the location of back pain and other musculoskeletal pain; personal and family history of known axSpA manifestations; and details of their IBD course, activity and treatment.

IBD patients, with back pain duration $>3$ months with onset before 45 years were considered to have a medium diagnostic probability (MDP) for axSpA. MDP-positive IBD patients were compared with MDP-negative IBD patients and logistic regression was used to model the association with clinical features.

Results: Four hundred and seventy consecutive IBD patients (mean age 54 years; $46 \%$ male) were surveyed. Two hundred and nine patients (59\%) replied, of whom 191 patients $(69 \%)$ consented to participate. One hundred and seventy-three $(91 \%)$ of those who consented had a valid completed questionnaire and were included for data analysis. Of these, $74 \%$ had Ulcerative Colitis and $26 \%$ had Crohn's disease. Their mean age was 58 years, $39 \%$ male. Mean age at IBD diagnosis was 39 years, mean IBD disease duration 19 yrs. CBP (back pain greater than three months) was reported by $76 \%$. Inflammatory back pain fulfilling Calin, Berlin, ASAS criteria was seen in $23 \%, 29 \%$, and $15 \%$ respectively. In addition, $80 \%$ reported peripheral musculoskeletal pain. Self-reported personal history of enthesitis, reactive arthritis (ReA), acute anterior uveitis (AAU), skin psoriasis (PSO) and dactylitis were $50 \%, 30 \%, 24 \%, 15 \%$ and $0 \%$ respectively. Self-reported family history of IBD, ReA, PSO, axSpA and AAU were $60 \%, 36 \%, 22 \%, 11 \%$, and $1 \%$ respectively. Ninety-one $(53 \%)$ patients were MDP-positive and $82(47 \%)$ patients were MDP-negative. The clinical characteristics associated with MDP (adjusted for age at invitation) were: the presence of inflammatory back pain using ASAS criteria [OR $8.84(1.61,48.67) ; p=0.01]$, longer interval between symptom onset and gastroenterologist diagnosis of IBD [OR $1.09(1.03,1.16) ; p=0.005]$, and use of rectal topical 5-aminosalicylic acid [OR $3.27(1.11,9.68) ; p=0.03]$.

Conclusion: Chronic back pain and peripheral musculoskeletal pain are common in a secondary care IBD population. In IBD patients, with back pain duration $>3$ months and onset before 45 years, the presence of inflammatory back pain, longer diagnostic delay of IBD and the use of rectal topical 5-aminosalicylic acid were associated with a higher clinical probability of axSpA. The identification of these clinical features may not only improve the quality and specificity of Rheumatology referrals from Gastroenterology in this subgroup of patients but also lends real world evidence to current ASAS-endorsed recommendations for early referral of patients with a suspicion of axial spondyloarthritis.

Disclosure of Interests: Chong Seng Edwin Lim Grant/research support from: AbbVie - Research support/grant but NOT for this study., Mark Tremelling: None declared, Louise Hamilton: None declared, Alexander Macgregor: None declared, Karl Gaffney Grant/research support from: AbbVie, Celgene, MSD, Novartis, Pfizer, and UCB Pharma, Consultant of: AbbVie, Celgene, MSD, Novartis, Pfizer, and UCB Pharma, Speakers bureau: AbbVie, Celgene, MSD, Novartis, Pfizer, and UCB Pharma

DOI: 10.1136/annrheumdis-2020-eular.576

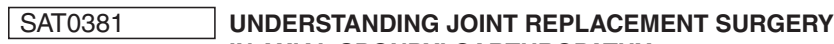 IN AXIAL SPONDYLOARTHROPATHY}

$\underline{\text { S. Maguire }}{ }^{1}$, P. Gallagher ${ }^{2}$, F. B. O'shea ${ }^{1} .^{1}$ St James Hospital, Rheumatology, Dublin, Ireland; ${ }^{2}$ St Vincents Hospital, Rheumatology, Dublin, Ireland

Background: Axial spondyloarthropathy $(\operatorname{axSpA})$ is a form of inflammatory arthritis that primarily affects the axial skeleton and sacroiliac joint, but can be associated 